\title{
NATIONAL ADVISORY COMMITTEE FOR AERONAUTICS
}

TECHNICAL NOTE 3596

ON THE PERMEABILITY OF POROUS MATERIALS

By E. Carson Yates, Jr.

Langley Aeronautical Laboratory

Langley Field, Va.
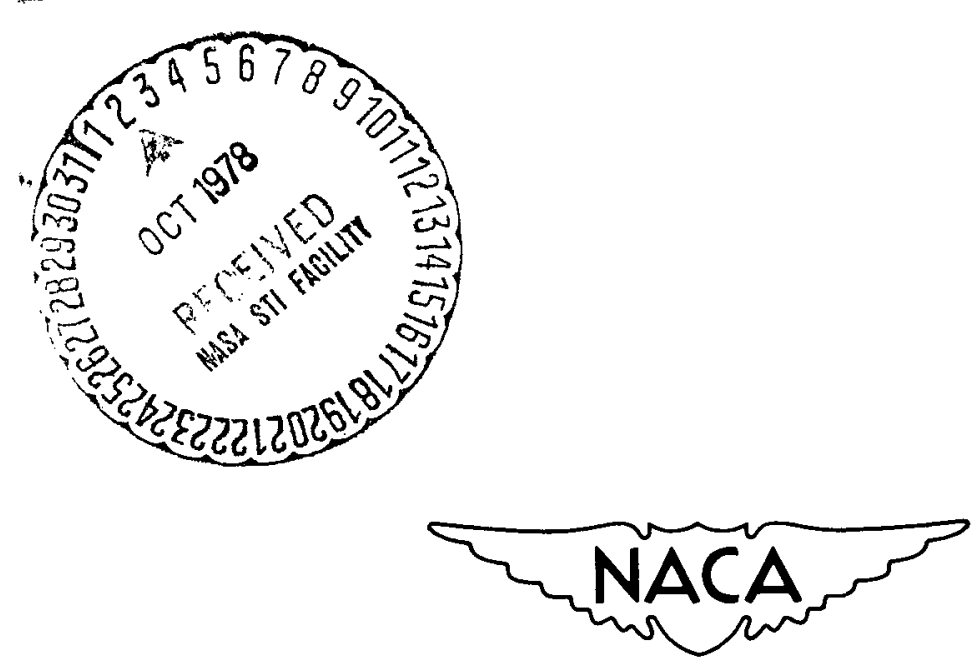

Washington

January 1956

(NASA-TM-79825) ON THE PERMEABIIITY OF POROOS MAIERIAIS (National Advisory Comittee for: $A \in$ ronautics, ${ }^{\circ} \mathrm{p}$
N78-78604

Onclas

$00 / 24 \quad \equiv 2228$ 
TECHWICAL NOTE 3596

ON THE PERMEABILITY OF POROUS MATERIALS

By E. Carson Yates, Jr.

\section{SUMMARY}

The effects on porous-material permeability characteristics of the absolute pressure level (and associated scale effects), choking of the flow, bending the material; and other factors have been investigated. Samples of rolled 30-by 250-mesh Dutch weave Monel metal cloth and 1/16-inch-thick sintered bronze were calibrated with constant upstream pressures of 1 atmosphere and $2 \frac{1}{3}$ atmospheres (varying downstream pressure) and with constant downstream pressure of 1 atmosphere (varying upstream pressure). Experiments showed permeability characteristics to be appreciably affected by absolute pressure level, flow choking, and thickness of the material. Moderate bending of the material caused no noticeable change in the permeability. Simple calculation and correlation procedures are presented for determining permeability characteristics with reasonable accuracy when experimental data are limited.

\section{INTRODUCTION}

Accurate and comprehensive information on the permeability characteristics of porous materials is essential to the proper design of areasuction installations on wings, flaps, inlets, and wind tunnels. It was found in the course of designing a wing using suction through a porous material that available calibration information was not sufficient for predicting permeability characteristics for the range of operating conditions (absolute pressure levels) anticipated. Since most previous calibrations (for example, refs. 1 and 2) were found to have been made with a single fixed relation existing between the Mach number and Reynolds number of the flow, the effect on permeability of independent variation of these quantities (caused, for example, by differences of absolute pressure level) could not be evaluated. For example, if a calibration test is made with the upstream pressure held constant and the downstream pressure varied, it can easily be shown that the ratio of Reynolds number to Mach number (both based on upstream conditions) is a constant. In addition, previous investigations were found to include no information regarding choking of the flow following the occurrence of sonic velocity within the pores, bending the material, and, in the case of wire cloth, rolling the material. 
The need for information on the aforementioned factors is emphasized by the following considerations. For a given pressure difference across a porous material, scale effects associated with absolute pressure level were believed to influence flow velocities. If this influence is appreciable, it should be considered in the selection of a porous material. Consideration of the limitation on inflow velocity imposed by flow choking is important in some installations. For example, in wing-suction applications choking may be used advantageously to limit inflow over areas where large pressure differences exist, so that chordwise grading of permeability may be dispensed with. Bending the porous material could affect permeability by changing the sizes of openings in the material and by changing the flow from one dimensional to two dimensional. These changes could have significant effects, for example, in flow near the leading edge of a wing.

The investigation reported herein was undertaken to determine the influence on the permeability of porous material of the aforementioned factors. Representative samples of wire cloth and sintered bronze (two samples of each) were calibrated by holding the upstream pressure constant at 1 atmosphere and varying the downstream pressure, by holding the upstream pressure constant at $2 \frac{1}{3}$ atmospheres and varying the downstream pressure, and by holding the downstream pressure constant at one atmosphere and varying the upstream pressure. Testing in this manner yielded independent variation of Mach number and Reynolds number. The analysis includes comparison of the wire-cloth calibrations with a calibration for the same material bent to form the leading edge of a wing model which was tested in the Langley 19-foot pressure tunnel. Data obtained in the

Langley cascade aerodynamics laboratory for the wire cloth rolled to various thicknesses are also shown. The analysis indicates methods for calculating or estimating permeability calibration curves, and these methods are presented herein.

The possible large effect on permeability of the presence of a component of the inlet velocity which is parallel to the porous surface (as discussed in ref. 3) is not considered herein. However, since the pores in the materials investigated are very much smaller than those in the perforated materials of reference 3, it is believed that the influence of a parallel flow component in the present case would be considerably less than that reported in reference 3 .

\section{SYMBOLS}

A cross-sectional area of venturi throat

D effective diameter of pores, ft

g gravitational acceleration, $\mathrm{ft} / \mathrm{sec}^{2}$ 


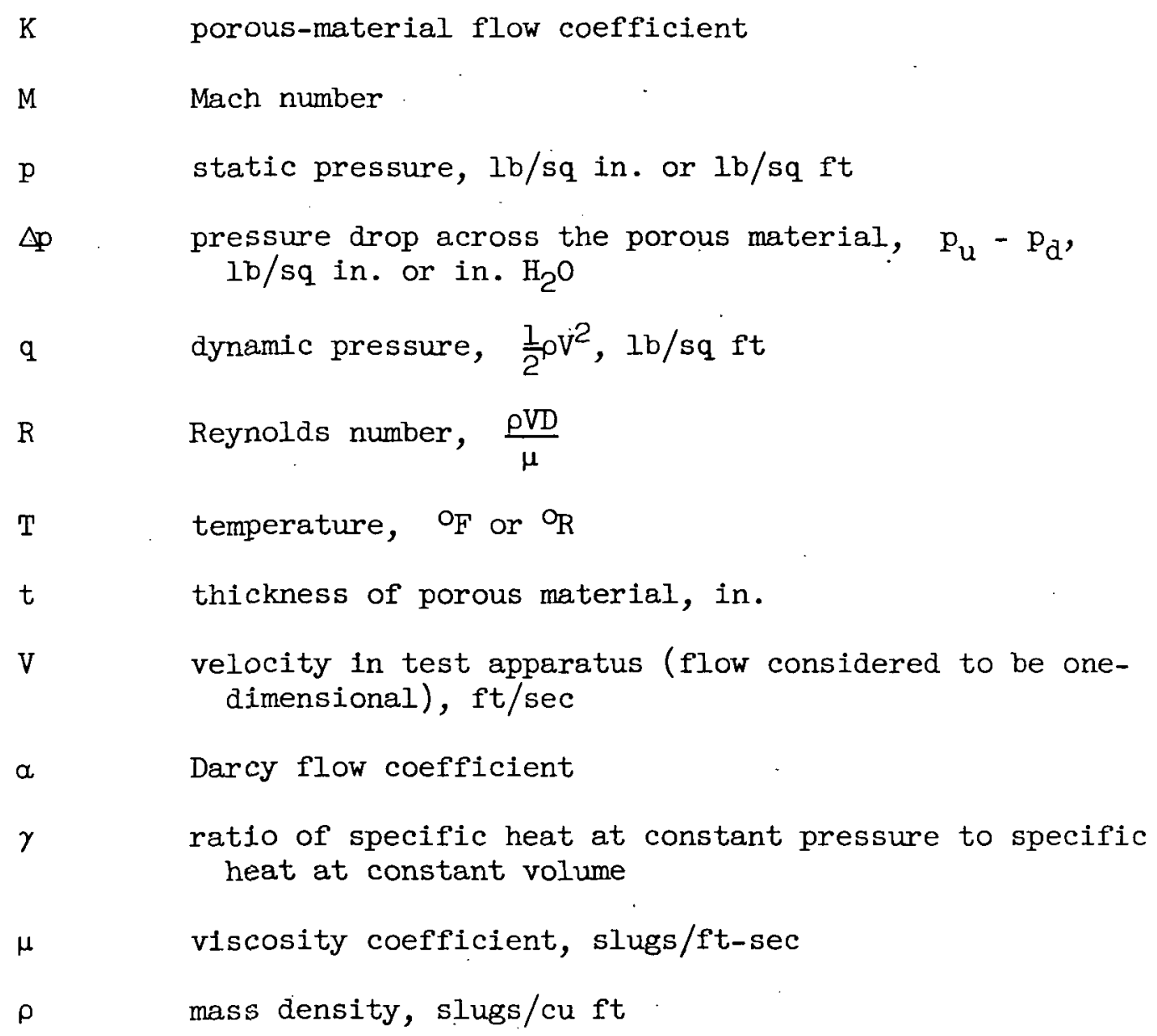

Subscripts :

d station just downstream of the porous material

$\mathrm{t} \quad$ station at the venturi throat

u station just upstream of the porous material

\section{DEFINITIONS}

The term "porosity" as used herein is defined as the percentage of void present in the porous material.

Permeability is a qualitative term related to the resistance of the material to fluid flow. The greater the resistance, the less, the permeability, and vice versa. 


\section{APPARATUS AND TECHNIQUE}

\section{APPARATUS}

The apparatus used in the tests with constant upstream pressure is shown schematically in figure 1 . It consisted of a 2-inch insidediameter pipe with a flange, clamp plate, and gaskets at one end to hold the sample and a wooden venturi at the other end. Downstream of the $\frac{1}{2}$ inch-diameter-venturi throat was an exhaust pipe containing a gate valve for regulating the downstream pressure $p_{d}$.

The apparatus used in the tests with varying upstream pressure is shown in figure 2. This apparatus was the same as that used for constant upstream pressure except that an additional length of 2-inch pipe was attached upstream of the sample, and the downstream pipe and valve were moved upstream of the entire setup. Fine wire screens were placed 2 diameters upstream of the sample to help maintain flow uniformity. High and low pressure sources used were the Langley 19-foot pressure tunnel and the suction side of a centrifugal compressor.

Downstream flow velocities were determined by applying onedimensional-flow relations between stations $d$ and $t$ (figs. 1 and 2) as follows:

$$
v_{d}=\sqrt{\frac{2\left(p_{d}-p_{t}\right)}{\rho_{d}\left[\left(\frac{A_{d}}{A_{t}}\right)^{2}-1\right]}}
$$

Continuity and isothermal conditions were used to relate stations $u$ and $d$.

\section{MATERIAL}

Two flat samples of 30-by 250-mesh Dutch weave Monel metal wire cloth were calibrated. The wire diameters for these samples were 0.0080 inch for the wires that were 30 per inch and 0.0040 inch for the wires that were 250 per inch. The samples were rolled to a thickness of 0.017 inch from an original thickness of 0.026 inch. For purposes of this investigation, tests of wire cloth of other mesh dimensions were not made because examination of data available from the Langley cascade aerodynamics laboratory indicated that other meshes would have generally 
similar flow characteristics. Results presented herein for the wire cloth are, therefore, considered to be typical. Inasmuch as the two wire-cloth samples are ostensibly identical, data are shown for only one of them. However, a 6-percent difference occurred between the flow velocities for the two samples at a given value of $\triangle p$. This difference gives some indication of the variation of permeability which may occur for a given thickness of wire cloth.

In addition to the calibrations of the wire cloth, calibrations were also made of two flat samples of $\frac{1}{16}$ inch-thick sintered bronze. A theoretical and experimental investigation of the permeability of various sintered metals is reported in reference 4.

PROCEDURE

In the calibrations made for this investigation, three methods of pressure variation were used. The first method employed a pressure $p_{u}$ upstream of the sample of approximately 1 atmosphere and a variable suction pressure $p_{d}$ downstream; the second method employed $p_{u}$ of approximately $2 \frac{1}{3}$ atmospheres with a variable $\mathrm{p}_{\mathrm{d}}$; and the third method employed a variable $p_{u}$ with $p_{d}$ of 1 atmosphere. In every case a 2-inch-diameter sample of porous material was open to the flow, and the results presented represent an average of conditions over the sample. Before each calibration the sample was thoroughly cleaned with acetone.

The pressures indicated by the manometers shown in figures 1 and 2 were recorded for various values of $\Delta p$. Reference temperature and pressure were also recorded. For each value of $\Delta$, the flow velocities were obtained from the calibration equation (eq. 1).

\section{ACCURACY}

The repeatability of the' wire-cloth calibrations was determined by making several duplicate calibrations. The results indicated that the calibrations were repeatable within about 1 percent. No repeatability check was made for the sintered materials. The venturi calibration was based on the assumption of incompressible flow in the venturi. This assumption resulted in a maximum error of about 1 percent at the highest velocities obtained. Calculations indicate that errors in the velocities caused by venturi scale effect were less than 0.6 percent. 


\section{RESULTS AND DISCUSSION}

The basic permeability data are presented as plots of $v_{u}$ against $\triangle \mathrm{p}$, inasmuch as the quantity desired for boundary-layer-suction applications is inflow velocity for a given pressure drop. Also, since the porous-material flow phenomena depend only on Mach number and Reynolds number, any dimensionless flow characteristic (for example, $\Delta p / p u$ or $\Delta p / q_{u}$ ) plotted as a function of $M$ and $R$ will completely describe the flow. For purposes of analysis, plots of this type are used herein. The particular form chosen for this plot for the wire cloth is $\Delta p / q_{u}$ as a function of $M_{u}$ for various values of the parameter $R_{u} / D_{u_{u}}$. The quotient $R_{U} / D_{u}$ was chosen because this quantity is constant for calibrations with constant $\mathrm{p}_{\mathrm{u}}$ and $\mathrm{T}_{\mathrm{u}}$. Since the length which appears in the expression for $R$ is significant only in comparisons between different materials, and since only a single wire cloth is considered, this length has been eliminated from the Reynolds number by using the quantity $R_{U} / D$ instead of $R_{u}$. For the sintered materials the Mach number effects were expected to be small, and $\Delta \mathrm{p} / \mathrm{q}_{\mathrm{u}}$ is therefore presented as a function of $R$ for various values of $R_{u} / D_{u_{u}}$.

\section{WIRE CLOTH}

Effect of Mach Number and Reynolds Number on Permeability

The differences between the calibration curves obtained by testing with the three upstream-pressure conditions described previously are shown in figure 3 . For the tests with $p_{u}$ variable, the downstream pressure $p_{d}$ was atmospheric, and at small values of $\Delta_{p}$ (valve nearly closed) the upstream pressure $p_{u}$ was only slightly greater than atmospheric. This condition is very nearly the same as that which existed at low values of $\triangle p$ for the tests at $p_{u}=1$ atmosphere, that is, $p_{u}$ of 1 atmosphere and $p_{d}$ slightly less than 1 atmosphere. Therefore, the curves for $p_{u}=1$ atmosphere and $p_{u}$ variable would be expectéd to lie very close together at small values of $\triangle$. By similar reasoning the curves for $p_{u}=2 \frac{1}{3}$ atmospheres and $p_{u}$ variable would be expected to lie very close together at large values of $\Delta p$. This behavior of the experimental data is shown in figure 3 .

The data of figure 3 are shown in figure $4^{\circ}$ in the form of $\triangle p / q_{u}$ as a function of $M_{u}$ with $R_{u} / D_{u}$ as a parameter. It is emphasized 
that this plot completely describes the flow for the given material regardless of the differences in pressure magnitudes or temperature. Any other nondimensional presentation of the data may consequently be derived from this figure, and any correlation of the data must be consistent with these results. In addition to the curves for $p_{u}=1$ atmosphere, $p_{u}=2 \frac{1}{3}$ atmospheres, and $p_{u}$ variable shown in figure 4 , contours are also shown for intermediate conditions of constant $p_{u}$ (and hence constant $\mathrm{R}_{\mathrm{U}} / \mathrm{DM}_{\mathrm{U}}$ ). These contours, obtained from cross plots of the three experimental curves, give complete calibration information over the entire Mach number and Reynolds number range covered by the tests. At low Reynolds numbers (upper left portion of the contours of fig. 4), the relatively large spread between the contours indicates that sizeable scale effects occur in this range. The rapidiy decreasing slopes of these portions of the curves of $\Delta / q_{u}$ as a function of $M_{u}$ are indicative of transition from creep flow $\left(\frac{\Delta p}{q_{u}} \sim \frac{1}{V}\right)$ to inertial flow $\left(\frac{\triangle p}{q_{u}}=\right.$ Constant). By comparison, scale effects are relatively small in the range of moderate and high values of $R_{u} / D M_{u}$ (center and right portions of $\mathrm{fig}$. 4). The upward turn of the contours as $\mathrm{M}_{u}$ increases is evidence of incipient choking.

Representation of a single calibration curve by $\frac{\Delta p}{q_{u}}=$ Constant.-

Figure 4 shows that above the range of creep flow and below the range of choked flow the experimental calibrations may be approximated by lines of $\frac{\triangle p}{q_{u}}=$ Constant. For $p_{u}=1$ atmosphere (fig. 4), the experimental $\frac{\Delta p}{q_{u}}$ curve deviates from the mean value by as much as 6 percent for values of $\mathrm{M}_{\mathrm{u}}$ between $1.6 \times 10^{-3}$ and $4.0 \times 10^{-3}$. However, solving $\frac{\Delta p}{\mathrm{q}_{u}}=$ Constant for the velocity will give values of $v_{u}$ which deviate from the experimental values by less than 3 percent for $1.6 \times 10^{-3}<\mathrm{M}_{\mathrm{u}}<4.0 \times 10^{-3}$, as is shown in figure 5. Thus for a particular calibration curve,

$$
V_{u}{ }^{*}=K \sqrt{\frac{2}{\rho_{u}}} \sqrt{\triangle p}
$$

where the constant is related to the orifice coefficient. A slightly modified form of this "orifice flow" equation was used in reference 5 to investigate the efficiency of cowlings. 
Equation (2) may be compared with the empirical equation $\Delta p=$ Constant $\times\left(v_{u}\right) \phi$ used in reference 6 . Calibrations of several porous materials and combinations of porous materials with $\mathrm{p}_{u}=1$ atmosphere indicate that $1<\phi<2$, where the lower value corresponds to purely viscous (creep) flow and the higher value corresponds to purely inertial flow.

Rough correlation of calibrations from $v_{u} \approx f\left(\frac{\Delta p}{p_{u}}\right)$ - - Based on

figure 4 a correlation procedure can be developed which will permit the use of available wire-cloth calibration data (for example, curves of $v_{u}$ as a function of $\Delta p$, for $p_{u}=1$ atmosphere) for design purposes at absolute pressure levels other than the calibration pressure level.

Figure 4 shows that for a given $\mathrm{M}_{u}$ (and hence $v_{u}$ ) in the range of-hi-gh-and-moderately_high_values_of $R_{u},-\Delta p / q_{u}$ _does_not vary_greatly with changes of $R_{u}$. An increase in $R_{u}$ of 125 percent yields a deçease in $\Delta p / q_{u}$ of about 20 percent. Now if the ordinate of figure 4 is multiplied by $\frac{\gamma}{2} M_{u}{ }^{2}$, the resulting coordinate $\Delta p / p_{u}$ will also decrease by 20 percent at a given $\mathrm{M}_{\mathrm{u}}$ (or, approximately, at a given $\mathrm{v}_{\mathrm{u}}$ ). It is seen from equation (2) that for constant $p_{\mathfrak{u}}$. (and $T_{\mathfrak{u}}$ ), $\triangle p$ is proportional to $\mathrm{v}_{\mathrm{u}}{ }^{2}$, so that for a given $\Delta \mathrm{p} / \mathrm{p}_{\mathrm{u}} ; \mathrm{v}_{\mathrm{u}}$ will increase by about 10 percent as $R_{u}$ increases by 125 percent. Therefore, a rough correlation of the data at moderate and high velocities for the three upstream-pressure conditions may be obtained by presenting $v_{u}$ as a function of $\Delta \mathrm{p} / \mathrm{p}_{\mathrm{u}}$. Such a plot is shown in figure 6. This type of presentation is essentially the same as that used in reference 3 , where $v_{u}$ was given as a function of $\triangle \mathrm{p} / \mathrm{\rho}$. For the Reynolds number range of the present tests the correlation is rough, but for $\Delta \mathrm{p} / \mathrm{p}_{\mathrm{u}}$ greater than about 0.2 , any one of the curves would serve as a reasonable approximation to the other two. The difference remaining between the calibration curves of figure 6 is, of course, entirely due to scale effect. From figures 4 and 6 it is concluded that beyond the region of creep flow, calibrations for wire cloth are not greatly affected by variation of Reynolds number, at least in the range of pressures and Reynolds numbers covered by this investigation. However, the curves of figure 4 and the difference remaining between the calibrations of figure 6 show that scale effect is related to absolute pressure level. . Further discussion of scale effects in a theoretical and experimental study of the flow resistance of sintered metals may be found in reference 4 and in the section herein entitled "Sintered Metal." 
In the selection of wire cloth for a given installation, the plot of $v_{u}$ against $\triangle / p_{u}$ could be used (at least at the higher values of $v_{u}$ ) to obtain an initial estimate of permeability regardless of absolute pressure level because the velocity spread is little more than the 6-percent spread which occurred for the two ostensibly identical samples. In other words, for selecting a porous material whose resistance is primarily inertial, the effect on permeability of a difference between design and calibration absolute pressure levels may be approximately. taken into account simply by plotting the permeability curve as the variation of $v_{u}$ with $\triangle p / p_{u}$. An empirical attempt to correlate calibrations between different materials is given in references 7 and 8 .

The experimentally observed relation $q_{d}=f(\Delta p)$.- In the analysis of the calibration data it was observed that for all three upstream pressure conditions $\left(p_{u}=1\right.$ atmosphere, $p_{u}=2 \frac{1}{3}$ atmospheres, and $p_{u}$ variable), plotting $q_{\alpha}$ against $\triangle p$ (fig. 7) tends to bring the three sets of data into coincidence, at least until the curve for $p_{u}=1$ atmosphere diverges because of choking. The correlation shown in figure 7 is, Indeed, so good that a single curve may reasonably be faired through the data. Figure 7, however, might best be considered as an illustration of the fact that a particular correlation might be obtained for a particular material in a particular range of test conditions, even though such correlation may not have general applicability. In the section which follows entitled "Sintered Metal" is shown an even more striking correlation obtained for a sintered bronze, where tests of similar materials failed to show similar correlation. An occurrence of this sort shows that correlation based on anything but the fundamental variables, Mach number. and Reynolds number, should be used only with great caution.

Comparison of $v_{u}$ and $v_{d}$; effects of choking.- In the preparation or use of calibration data, it. is important to recognize that a considerable difference may exist between the magnitudes of $v_{u}$ and. $v_{d}$. Inasmuch as some calibrations have been presented without distinction between upstream and downstream velocities, figure 8 includes a comparison of $V_{u}$ and $V_{d}$ for 0.017 -inch and 0.018-inch wire cloth. The $V_{d}$ curves were calculated from the $V_{u}$ curves by considering the flow through the material to be isothermal:

In the range of small values of $\Delta p, V_{d}$ and $V_{u}$ are nearly equal. But as $\triangle p$ increases, the loss of total pressure becomes large, choking 
occurs in the pores, and $V_{d}$ increases very rapidly. Therefore, $V_{d}$ is not a good approximation to $v_{u}$ at large values of $\triangle$. Strictly speaking, after the flow in the pores is completely choked, $v_{u}$ remains constant for all values of $\Delta p$. However, figure 8 shows a continuing slight rise in $V_{u}$ which is believed to be caused by the presence of subsonic boundary layers on the wire elements. These boundary layers will permit some slight increase of upstream velocity with increasing pressure drop, even though the external flow regions are fully choked.

\section{Effect of Rolling the Material}

The effect of rolling on the permeability of metal filter cloth is shown in figure 9 as obtained from data taken in the Langley cascade aerodynamics laboratory. Rolling or hammering the material apparently has little effect on the form of the calibration curve but changes only the magnitude of the velocities. Each curve shown for the rolled material can be found from any one of the other curves by multiplying the ordinates by a constant factor.

Figure 10 is a cross plot of the data of figure 9. This plot and the other data examined indicate approximately linear variation of downstream velocity $v_{d}$ with thickness, at least over the range of thickmess presented. The scatter of some of the points of figure 10 is attributed to the fact that the thicknesses were determined with a precision no better than \pm 0.0001 inch. Differences between the permeabilitles of two samples of the same thickness were mentioned previously in the section entitled "Material". The relation between permeability and porosity is further discussed in reference 9, and a semiempirical relation between $\triangle \mathrm{p} / \mathrm{q}_{\mathrm{u}}$ and porosity is given in references 1 and 10 .

\section{Effect of Bending the Material}

Flgure 8 presents a comparison of $v_{u}$ and $V_{d}$ curves for flat and curved specimens of the 30- by 250-mesh filter cloth. The $v_{u}$ curve for the 0.017 -inch thickness is that of the present investigation. The $\mathrm{V}_{\mathrm{u}}$ curve for the 0.018 -inch thickness is the calibration for a porous leading edge as installed on a sweptback tapered wing model which was tested in the Langley 19-foot pressure tunnel. The leading-edge radius of the porous part of this wing varied from 0.540 inch to 0.307 inch, measured perpendicular to the leading edge. 
A comparison of these curves for $V_{u}$ indicates that the amount of bending present in the model leading edge caused no noticeable change in the form of the curve, although more extreme bending might possibly produce a change. The curve for the 0.018-inch material, can be computed from the curve for the 0.017-inch material by multiplying the ordinates by a constant factor. The magnitudes of the velocities are likewise unaffected by this amount of bending because the difference in magnitude between the two $v_{u}$ curves is accounted for by the difference in thickness of the two samples.

\section{SINTERED METAL}

Calibration curves are shown in figure 11 for two samples (designated $A$ and $B$ ) of sintered bronze similar to that used in references 6 , 11 , and 12. For comparison, the calibrations for the wire cloth (fig. 3) and for the sintered bronze of figure 7 of reference 13 are included.

The curves for samples A and B (fig. 1l) show a variation with absolute pressure level similar to that for the wire-cloth calibrations. This variation is not in agreement with the results shown from reference 13. The tests of reference 13 yielded the same calibration curve for $p_{u}$ of 1 atmosphere, for $p_{u}$ varying higher than 1 atmosphere, and for $p_{u}$ varying lower than $I$ atmosphere. In view of this difference of results each set of data, that is, for sample A, sample B, and the sample of reference 13, was examined for consistency within itself. This examination was made as follows:

The dimensionless quantity $\frac{p_{\mathfrak{u}}-p}{q}$ (Euler number) is in general a function of Mach number and Reynolds number. (See, for example, fig. 4.) For the sintered materials considered herein, it was anticipated that the flow resistance would be primarily viscous, so that $\frac{p_{u}-p}{q}$ would be predominantly a function of $R$. If Mach number effects are actually. negligible for a given porous material, then $\frac{p_{u}-p}{q}$ should have the same value for a given Reynolds number regardless of absolute pressure level. Since the flow is approximately isothermal, and since temperature differences between calibrations for each sample were not large (these conditions are assumed to apply also for ref. 13), $\frac{R \mu}{D}=\rho_{u} V_{u}$ is used Instead of $R$. In order to represent the flow in the pores, 
the quantity $\frac{p_{u}-p}{q}$ must be evaluated within the porous material. Since no measurements of conditions within the materials were available, the averages of upstream and downstream conditions were used. Thus, $\mathrm{p}=\frac{\mathrm{p}_{\mathrm{u}}+\mathrm{p}_{\mathrm{d}}}{2}$ and

$$
q=\frac{1}{2} \rho v^{2}=\frac{1}{2} \rho_{u} \frac{p}{p_{u}}\left(\frac{v_{u}+v_{d}}{2}\right)^{2}
$$

The quantity $\frac{p_{12}-p}{q}$ is plotted against $R \mu / D$ in figure 12 for samples $A$ and $B$ of the present investigation and for the data of reference 13 . It should be noted that this figure is analogous to figure 4 for the wire cloth.

Figure 12 shows that all three sets of data are consistent within themselves. The downward displacement of the points for $p_{u}=1$ atmosphere for sample $B$ is caused by compressibility effects since, for a given $R \mu / D$, this curve represents appreciably higher velocity within the material than the $p_{u}=2 \frac{1}{3}$ atmospheres and $p_{u}$ variable curves. The explanation for the insensitivity of the data of reference 13 to absolute pressure level is indicated by consideration of the slopes of the callbration curves. If the flow in the pores is completely viscous in nature, and if compressibility effects are negligibly small, the flow will be described by Polseuille's equation $V=k \frac{\Delta p}{\mu}$, where $k$ is constant for a given material. (See refs. 2 and 14.) Thus, in the range of very low velocities the sintered-metal calibration data would be expected to be independent of absolute pressure level.

The Polseuille equation is based on flow with constant density. As $\triangle p$ increased, the flow density change through the material became appreciable, and the flow became of the Darcy type. This type of flow may involve appreciable density change, but the flow resistance remains predominantly viscous. Correlation of the data on the basis of Darcy's equation

or

$$
p_{u}^{2}-p_{d}^{2}=\frac{2 g \mu t}{\alpha} \frac{p_{d}}{\rho_{d}} \rho_{u} v_{u}
$$

$$
\Delta \mathrm{p}\left(2-\frac{\Delta p}{p_{u}}\right)=\frac{2 g \mu t}{\alpha} v_{u}
$$


is shown in figure 13. It may be observed from figure 13 that, since the relation between mass flow $\rho_{u} V_{u}$ and "pressure-square difference" $p_{u}{ }^{2}-p_{d}^{2}$ is not exactly linear, the flow is not a pure Darcy flow. The relation between the Darcy equation and the Poiseuille equation is discussed in reference 15. Equation (3) shows that for the Darcy-type flow, curves of $v_{u}$ against $\triangle p$ should be affected by absolute pressure level.

As $\Delta p$ increased further, inertial resistance became evident. Increasing inertia forces caused the slope of the curves (fig. II) to decrease as velocity increased. (See also ref.2.) In this range of higher velocities, absolute pressure level should again affect the calibration curve. This condition may be illustrated as follows:

Reverting to upstream flow conditions and ignoring Mach number effects causes the general functional relation $\frac{\Delta p}{q}=f(M, R)$ to take
the form

$$
\frac{\triangleq p}{q_{u}}=f(R)
$$

In figure 14 this relation is shown to hold reasonably well up to choking. Curves of the type shown may be represented empirically by an equation of the form

$$
\frac{\Delta p}{q_{u}}=A R^{n-2}
$$

where $A$ and $n$ are in general variable. Substituting for $q_{u}$ and $R$ and rearranging gives

$$
\Delta \mathrm{p}=\frac{1}{2} A D^{\mathrm{n}-2} \mu^{2-n_{\rho_{u}}}{ }^{\mathrm{n}-1} V_{\mathrm{u}}{ }^{\mathrm{n}}
$$

or applying the equation of state gives

$$
\Delta P=B D^{n-2} T^{1-n_{\mu} 2-n_{p}} p_{u}^{n-1} v_{u}{ }^{n}
$$

where the gas constant has been absorbed into the parameter $B$. If $B$ is constant, for $n=1$, equations (6) and (7) reduce to the form of the Poiseuilie equation, and for $n=2$, they reduce to the inertial flow relation $\frac{\triangleq p}{q_{u}}=$ Constant. 
Since the curves of figure 14 may be approximated by straight lines over a wide range of $R \mu / D$, the parameters $B$ and $n$ in equation (7) may be considered constant, and the equation may then be used to indicate the relative extent to which $p_{u}$ affects the calibration. In the velocity range below about 5 feet per second, the data shown in figure 11 from reference 13 show $\triangle p$ varying with the 1.17 power of $v_{u}$. With this value being used as the value of $n$, it may easily be shown that only the 0.17 power of $p_{u}$ appears in the expression for $\Delta p$, and hence absolute pressure level affects the calibrations of reference 13 to the extent of only \pm 3 percent at $V_{u}=5$ feet per second. At higher velocities, $\mathrm{n}$ becomes greater, and the effect of absolute pressure level should be more noticeable. For comparison, below $v_{u}=5$ feet per second, sample A showed $\triangle p$ varying with the 1.26 power of $v_{u}$ for $\mathrm{p}_{\mathrm{u}}=1$ atmosphere and with the 1.33 power of $v_{u}$ for $p_{u}=2 \frac{1}{3}$ atmospheres.

At a given velocity, if the magnitude of pressure (and hence density) is increased, dynamic pressures are increased, and so the inertial resistance of the material is increased. This increased resistance tends to shift that portion of the permeability curve (fig. ll) to the right. The increase of density, however, increases the Reynolds number and hence decreases the $\Delta p$ required for a given dynamic pressure (fig. 12). This condition tends to shift the permeability curve to the left. It is believed that these opposing effects coincidentally cancel each other for the material of reference 13 .

It should be noted in passing that the occurrence of a linear relation between $\Delta$ and $V_{u}$ is not sufficient to indicate a Poiseuille flow. Equation (3) indicates that for the Darcy flow, $\Delta p$ will vary linearly with $v_{u}$. if $p_{u}$ is varied in such a way that $\frac{\Delta}{\bar{p}_{u}}=$ Constant. Also, for example, solving simultaneously the equations $\frac{\Delta p}{q_{u}}=$ Constant and $\triangle p=$ Constant $\times V_{u}$ indicates that linear variation of $\triangle p$ with $\mathrm{V}_{\mathrm{u}}$ may be obtained for isothermal flow with purely inertial resistance if $\mathrm{p}_{\mathrm{u}}$ is varied so that $\Delta \mathrm{p}=\frac{\text { Constant }}{\mathrm{p}_{\mathrm{u}}}$.

\section{CONCLUSIONS}

The permeability characteristics of representative samples of wire cloth and sintered metal have been investigated by means of flow callbration tests made over an independent range of Mach number and Reynolds number. The results and comparison with other data revealed the following information: 
1. Since the permeability characteristics of a given porous material depend only on Mach number and Reynolds number, values of absolute pressure and temperature affect the usual calibration curve of upstream velocity as a function of pressure drop by changing the Mach number and Reynolds number of the flow.

2. In the absence of complete experimental data, a rapid estimate of the variation of velocity with pressure drop for wire cloth may be obtained by assuming that the ratio of pressure drop to upstream dynamic pressure is a constant. The results may be expected to be reasonably accurate up to conditions where appreciable choking occurs in the pores.

3. For a given wire cloth, the spread between calibrations obtained by using different absolute pressure levels can be greatly reduced by plotting upstream velocity against the ratio of pressure drop to upstream pressure.

4. When the thickness of wire cloth is reduced by rolling or hammering, the velocity downstream of the material varies almost linearly with thickness for a given pressure drop, at least within the range of this study.

5. Variations of velocity with pressure drop for wire cloth are not noticeably changed by bending the material, at least within the range of this investigation.

Langley Aeronautical Laboratory, National Advisory Committee for Aeronautics, Langley Field, Va., October 7, 1955. 


\section{REFERENCES}

1. Eckert, B., and Pflüger, F.: The Resistance Coefficient of Commercial Round Wire Grids. NACA TM 1003, 1942.

2. Preston, J. H., and Rawcliffe, A. G.: Note on Sintered Metal With a View to Its Use as a Porous Surface in Distributed Suction Experiments. Tech. Rep. 9383, British A.R.C., Feb. 9, 1946.

3. Stokes, George M., Davis, Don D., Jr., and Sellers, Thomas B.: An Experimental Study of Porosity Characteristics of Perforated Materials in Normal and Parallel Flow. NACA TN 3085, 1954. (Supersedes NACA RM L53H07.)

4. Green, Leon, Jr., and Duwez, Pol: Fluid Flow Through Porous Metals. Jour. Appl. Mech.; vol. 18, no. 1, Mar. 1951, pp. 39-45.

5. Theodorsen, Theodore, Brevoort, M. J., and Stickle, George W.: Full-Scale Tests of N.A.C.A. Cowlings. NACA Rep. 592, 1937.

6. Dannenberg, Robert E., and Weiberg, James A.: Section Characteristics of a 10.5-Percent-Thick Airfoil With Area Suction As Affected by Chordwise Distribution of Permeability. NACA TN 2847, 1952.

7. Grootenhuis, P.: A Correlation of the Resistance to Air Flow of Wire Gauzes. The Chartered Mech. Engineer, vol. 1, no. 5, May 1954, pp. 253-254.

8. Grootenhuis, P.: The Flow of Gases Through Porous Metal Compacts. Engineering, vol. 167, no. 4340, Apr. 1, 1949, pp. 291-292.

9. Taylor, G. I., and Davies, R. M.: The Aerodynamics of Porous Sheets. R. \& M. No. 2237, British A.R.C., Apr. 1944 .

10. Annand, W. J. D.: The Resistance to Air Flow of Wire Gauzes. Jour. R.A.S., vol. 57, no. 507, Mar. 1953, pp. 141-146.

11. Nuber, Robert J., and Needham, James R., Jr.: Exploratory WindTunnel Investigation of the Effectiveness of Area Suction in Eliminating Leading-Edge Separation Over an NACA $64{ }_{1}$ A212 Airfoil. NACA TN 1741, 1948.

12. Braslow, Albert L., Burrows, Dale L., Tetervin, Neal, and Visconti, Fioravante: Experimental and Theoretical Studies of Area Suction for the Control of the Laminar Boundary Layer on an NACA 64A010 Airfoil. NACA Rep. 1025, 1951. (Supersedes NACA TN 1905 by Burrows, Braslow, and Tetervin and NACA TN 2112 by Braslow and Visconti.) 
13. Dannenberg, Robert E., Weiberg, James A., and Gambucci, Bruno J:: The Resistance to Air Flow of Porous Materials Suitable for Boundary-Layer-Control Applications Using Area Suction. NACA TN 3094, 1954.

14. Wilson, L. H., Sibbitt, W. L., and Jakob, M.: Flow of Gases in Porous Media. Jour. Appl. Phys., vol. 22, no. 8, Aug. 1951, pp. 1027-1030.

15. Muskat, M.: The Flow of Homogeneous Fluids Through Porous Media. (With an Introductory Chapter by R. D. Wyckoff.) McGraw-Hill Book Co., New York, 1937. (Second Printing by J. W. Edwards, Inc., Ann Arbor, Mich., 1946.) , 


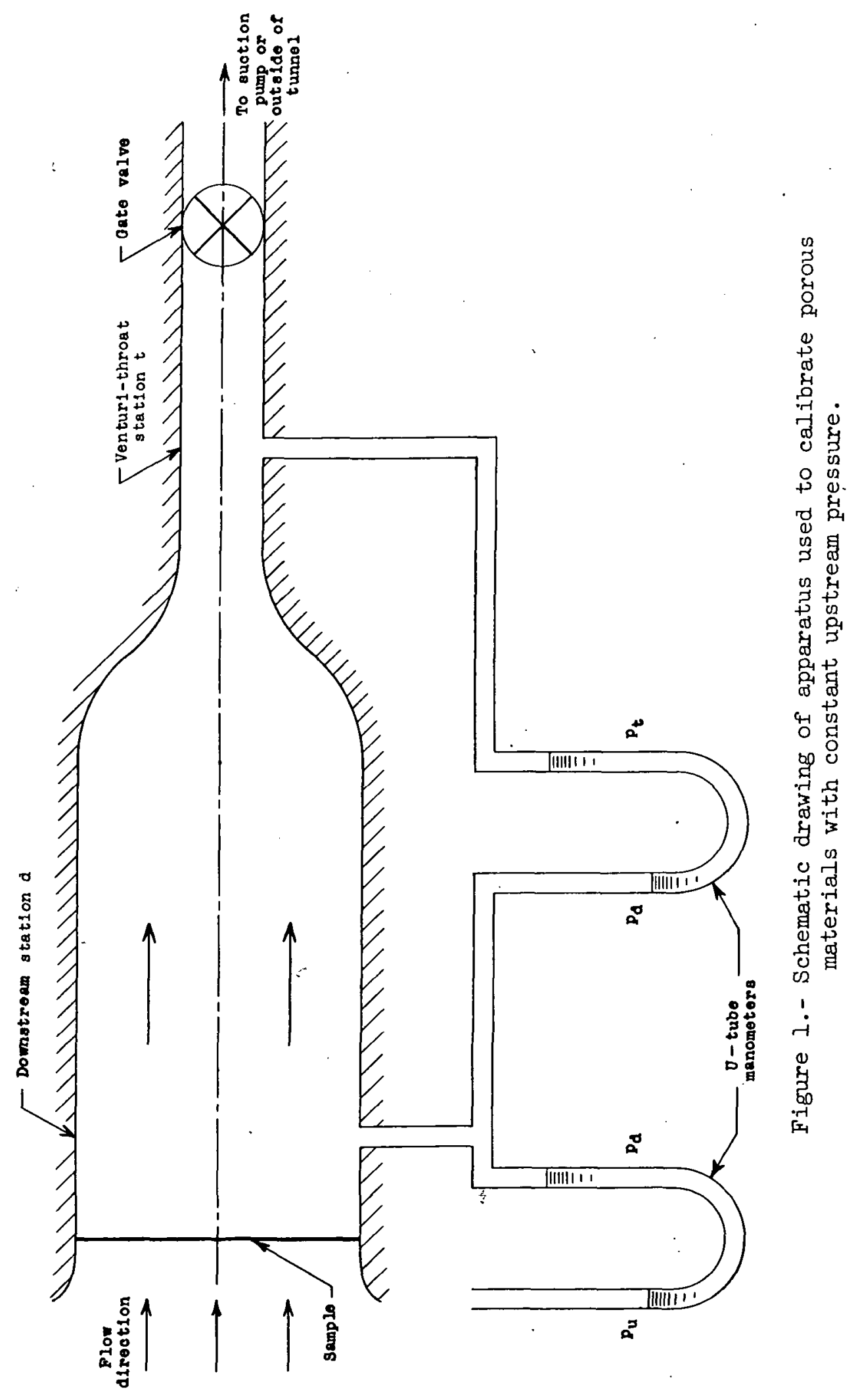




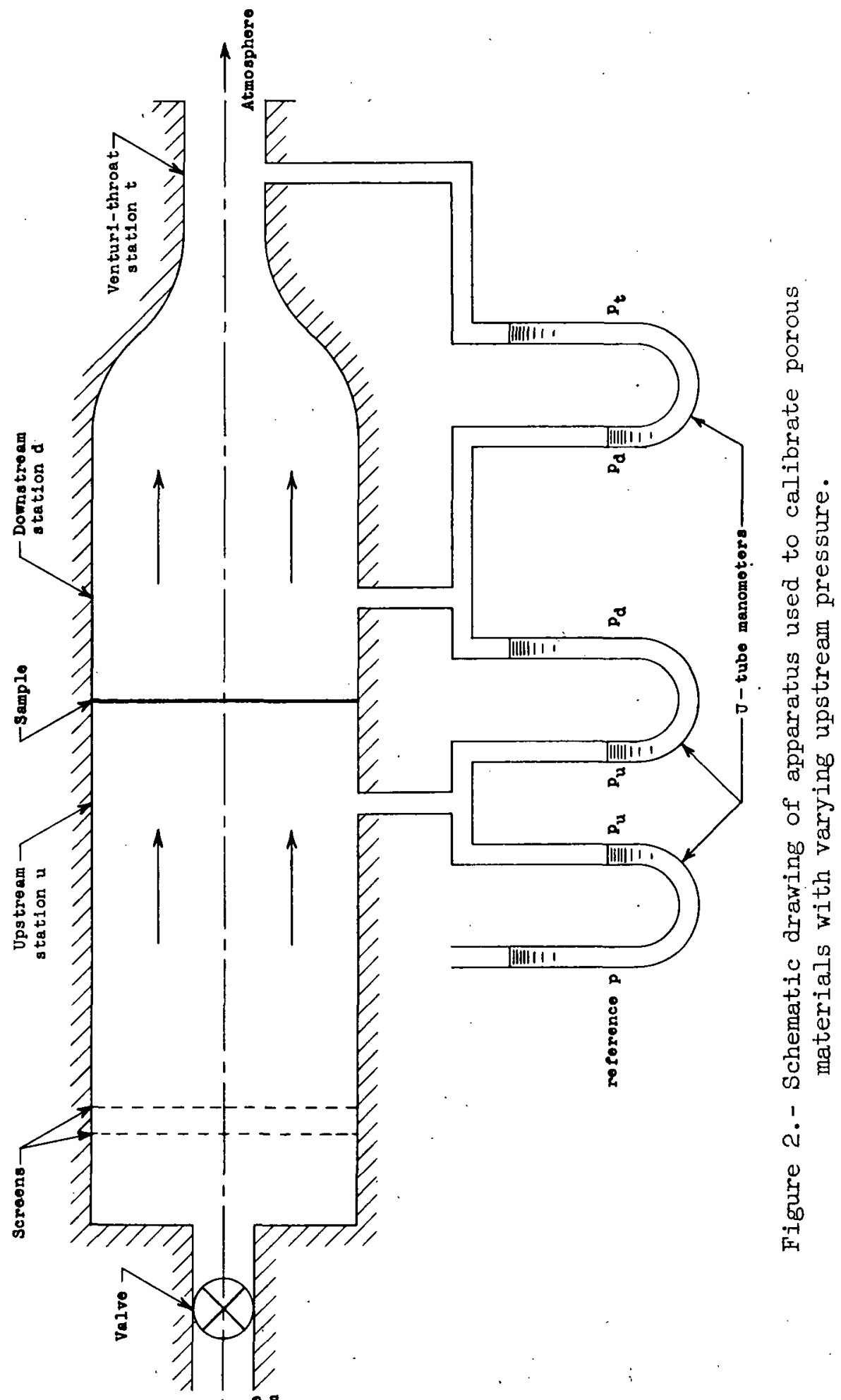

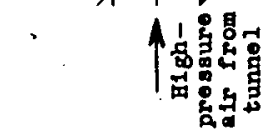




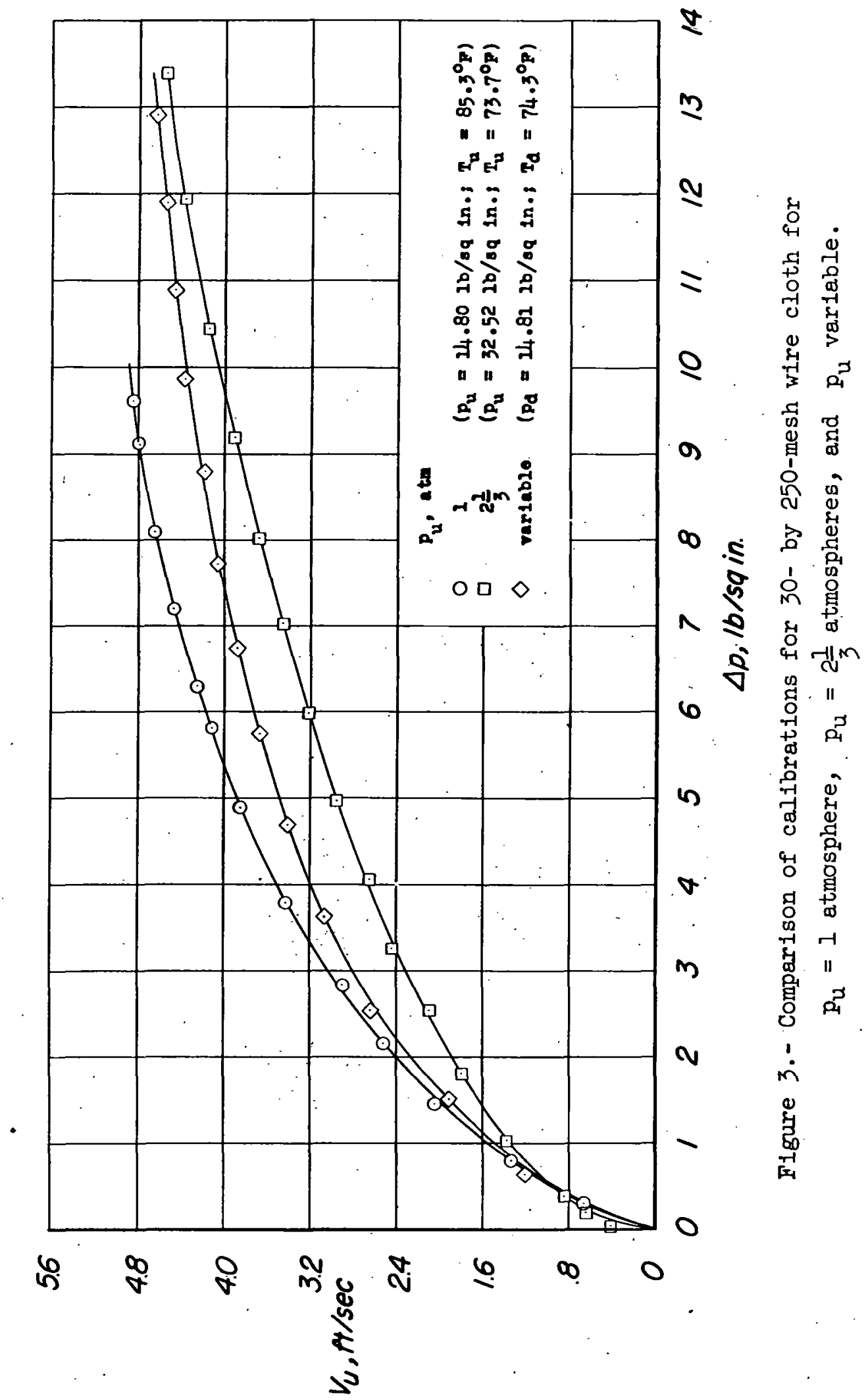




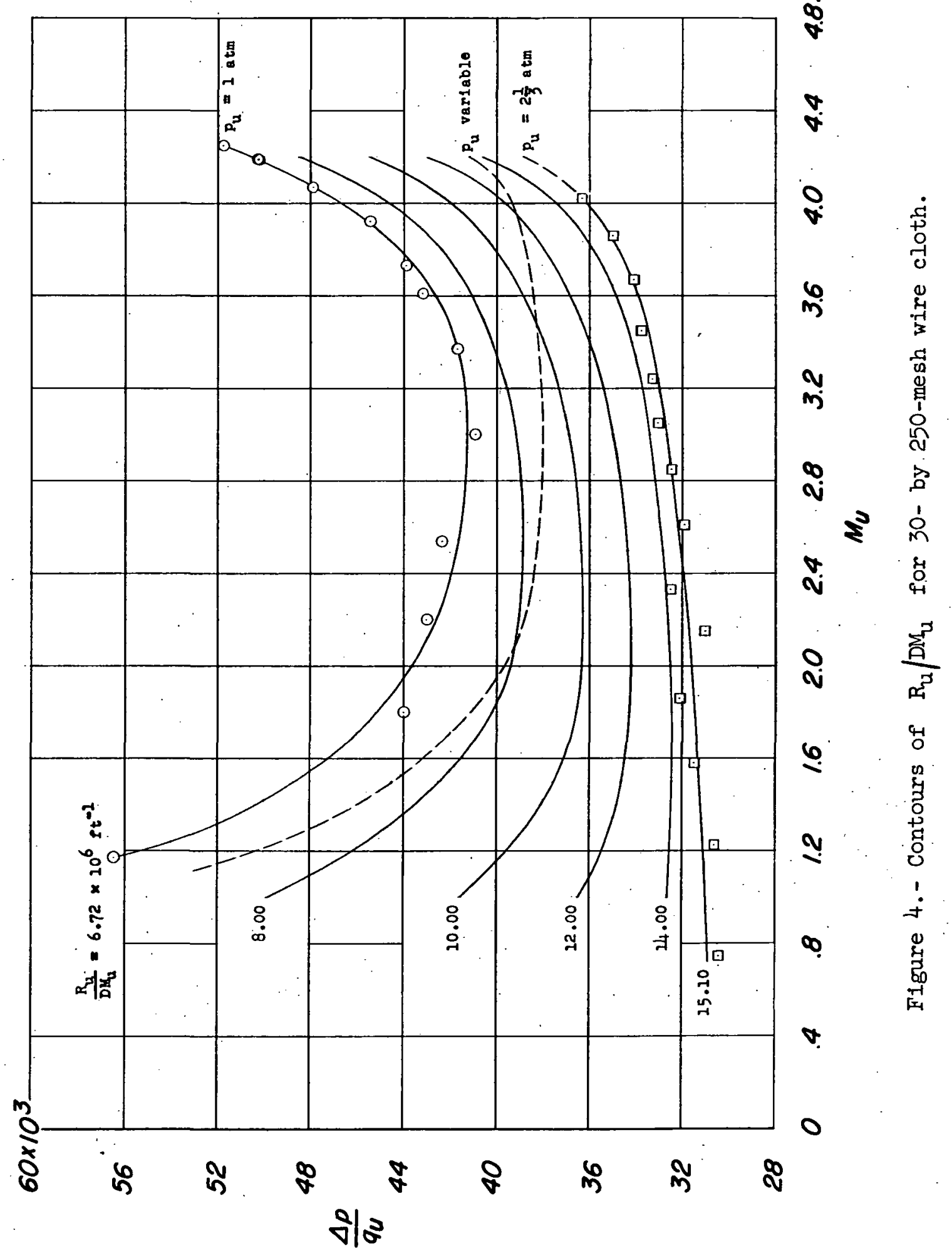




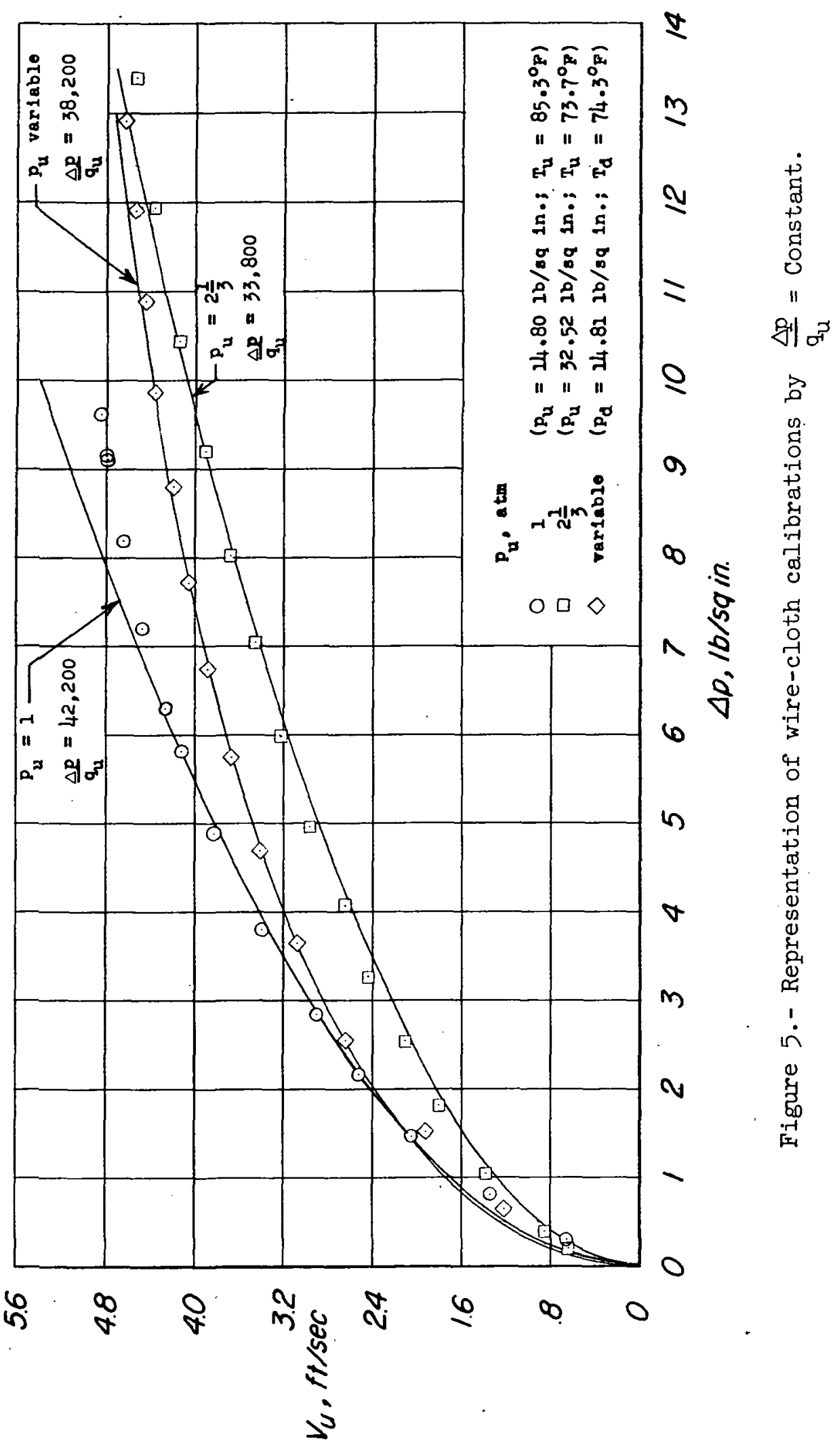




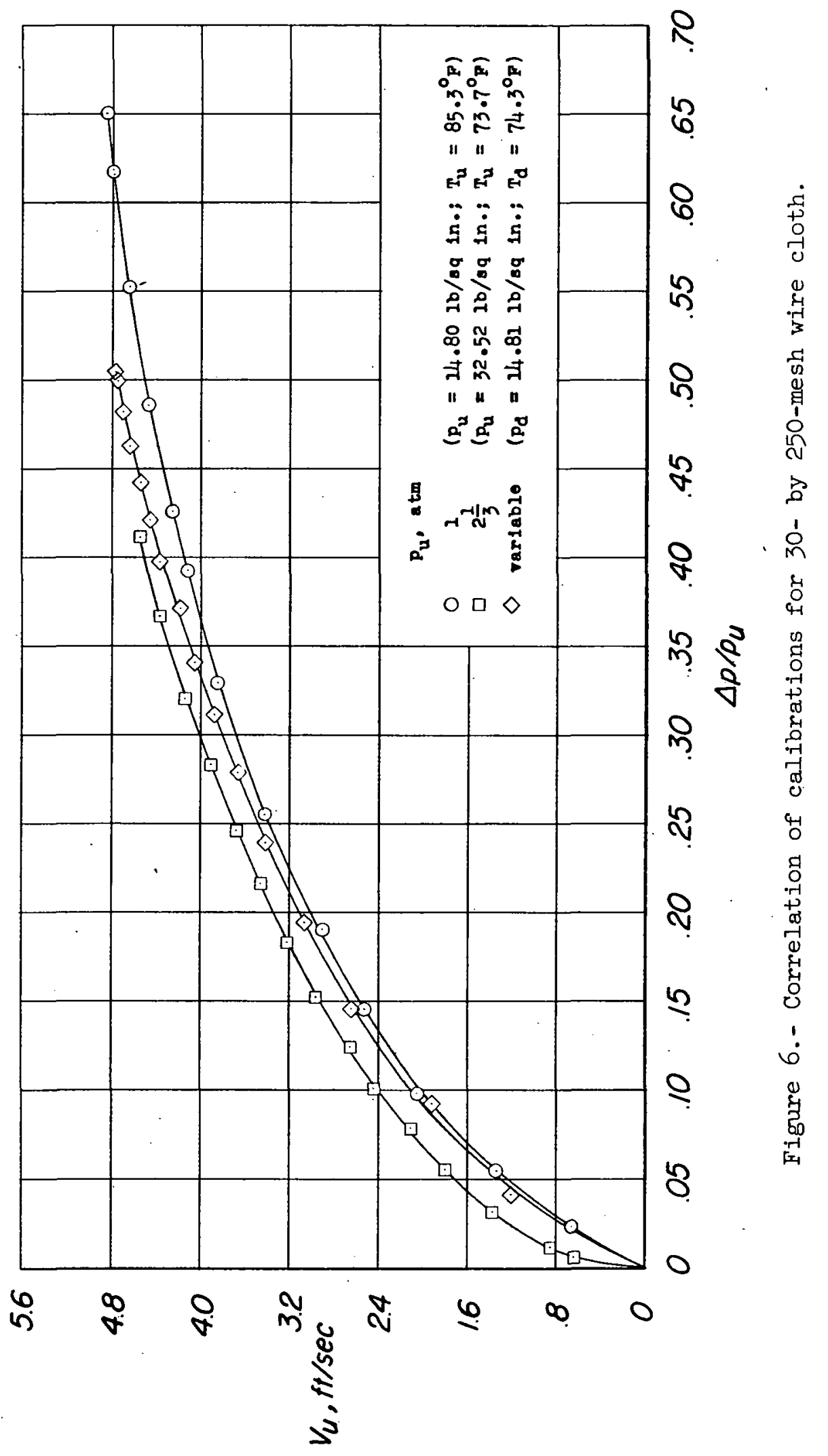




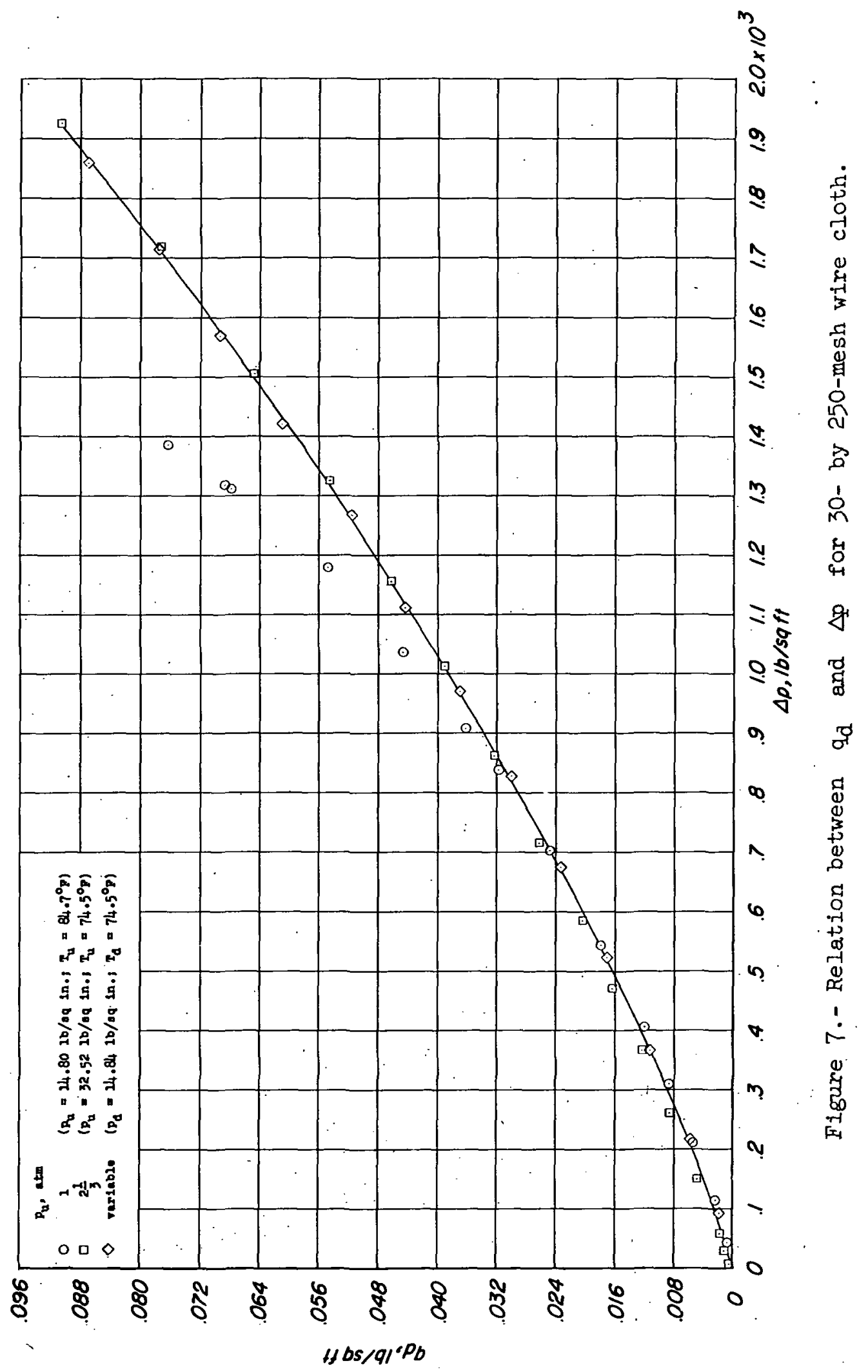




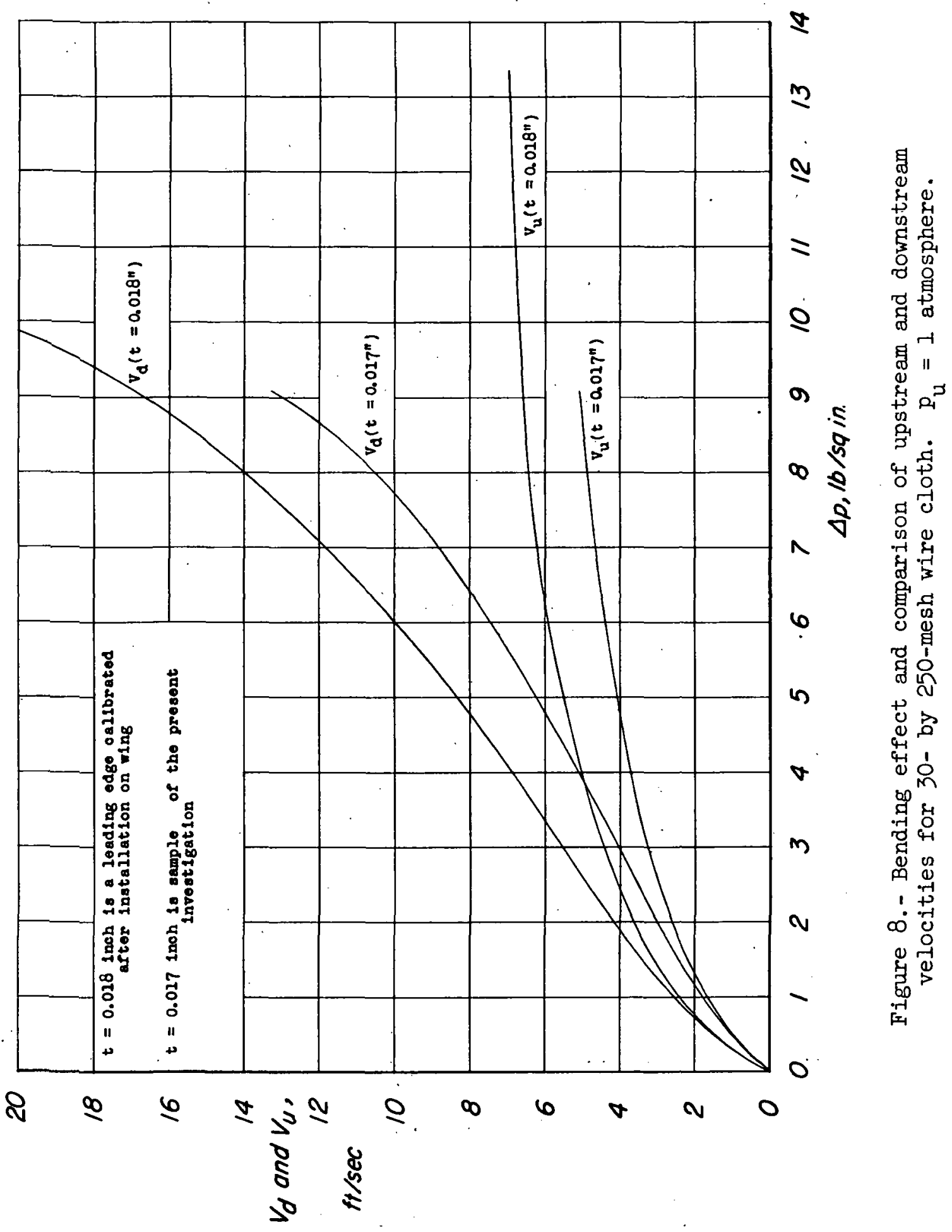




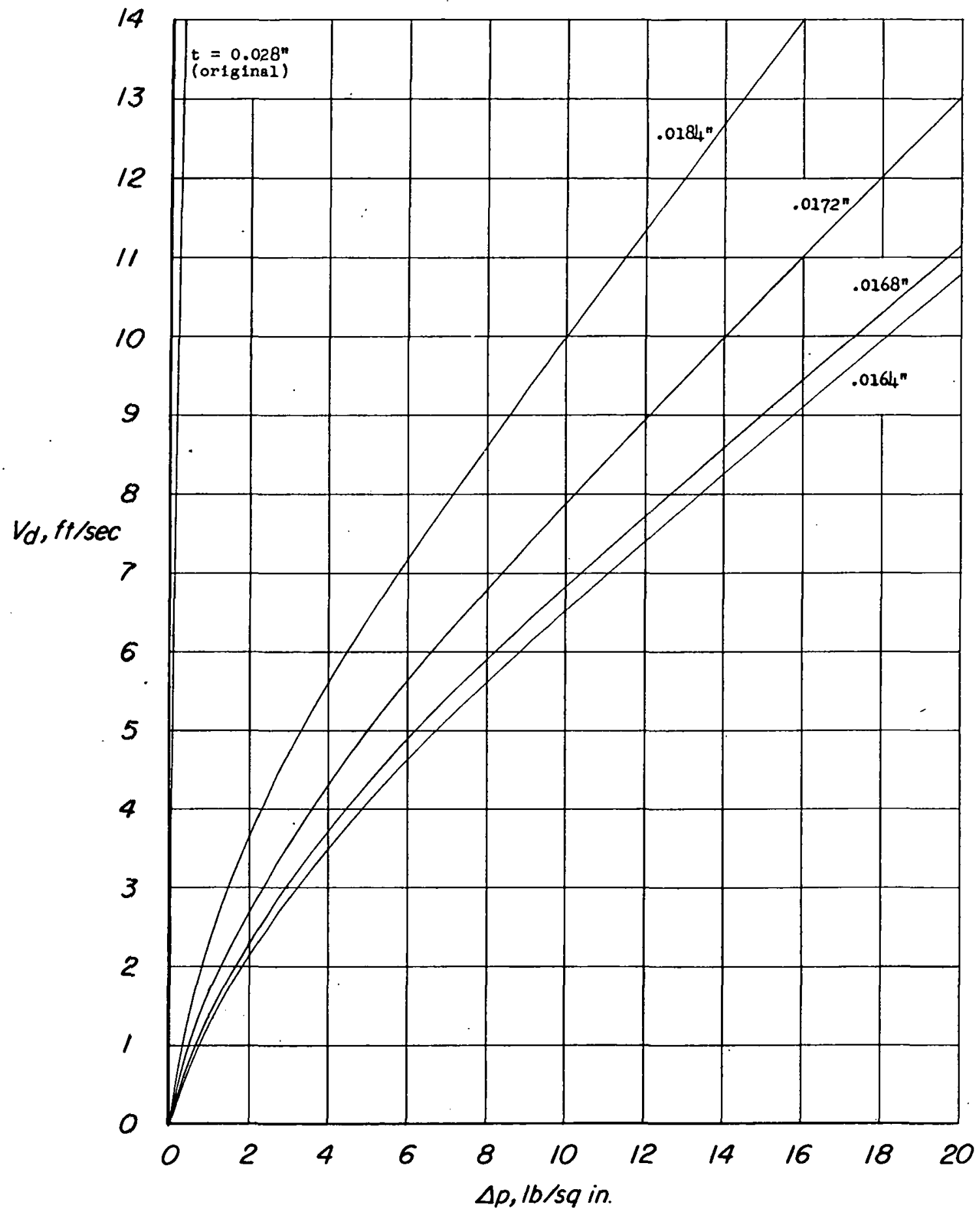

Figure 9.- Effect of rolling on permeability of 30- by 250-mesh Dutch weave Monel metal cloth. $p_{d}=1$ atmosphere, $p_{u}$ variable. 


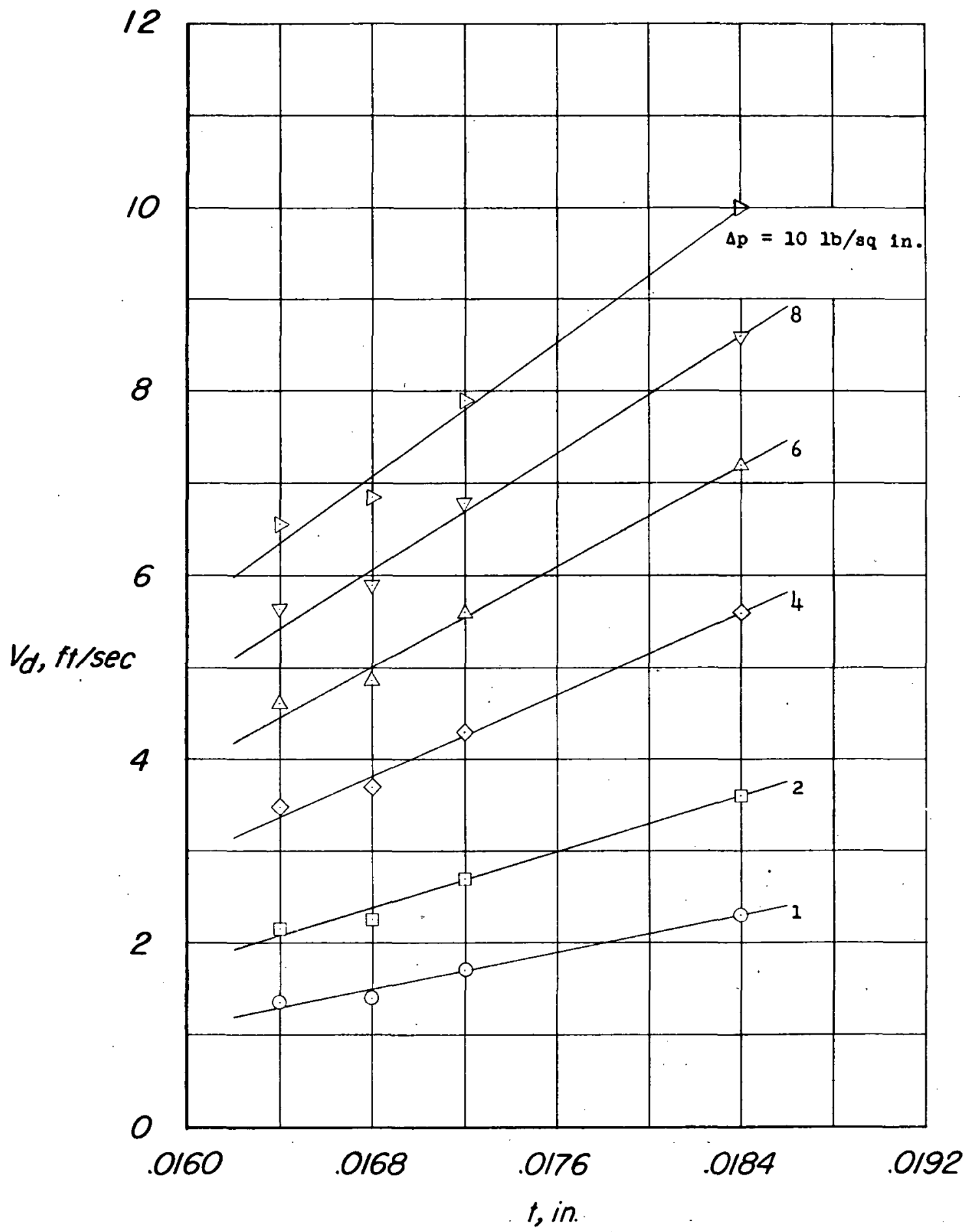

Figure 10.- Variation of permeability with thickness for 30- by 250-mesh wire cloth. Thickness reduced by rolling from original thickness of 0.028 inch. $\mathrm{p}_{\mathrm{d}}=1$ atmosphere, $\mathrm{p}_{\mathrm{u}}$ variable. 


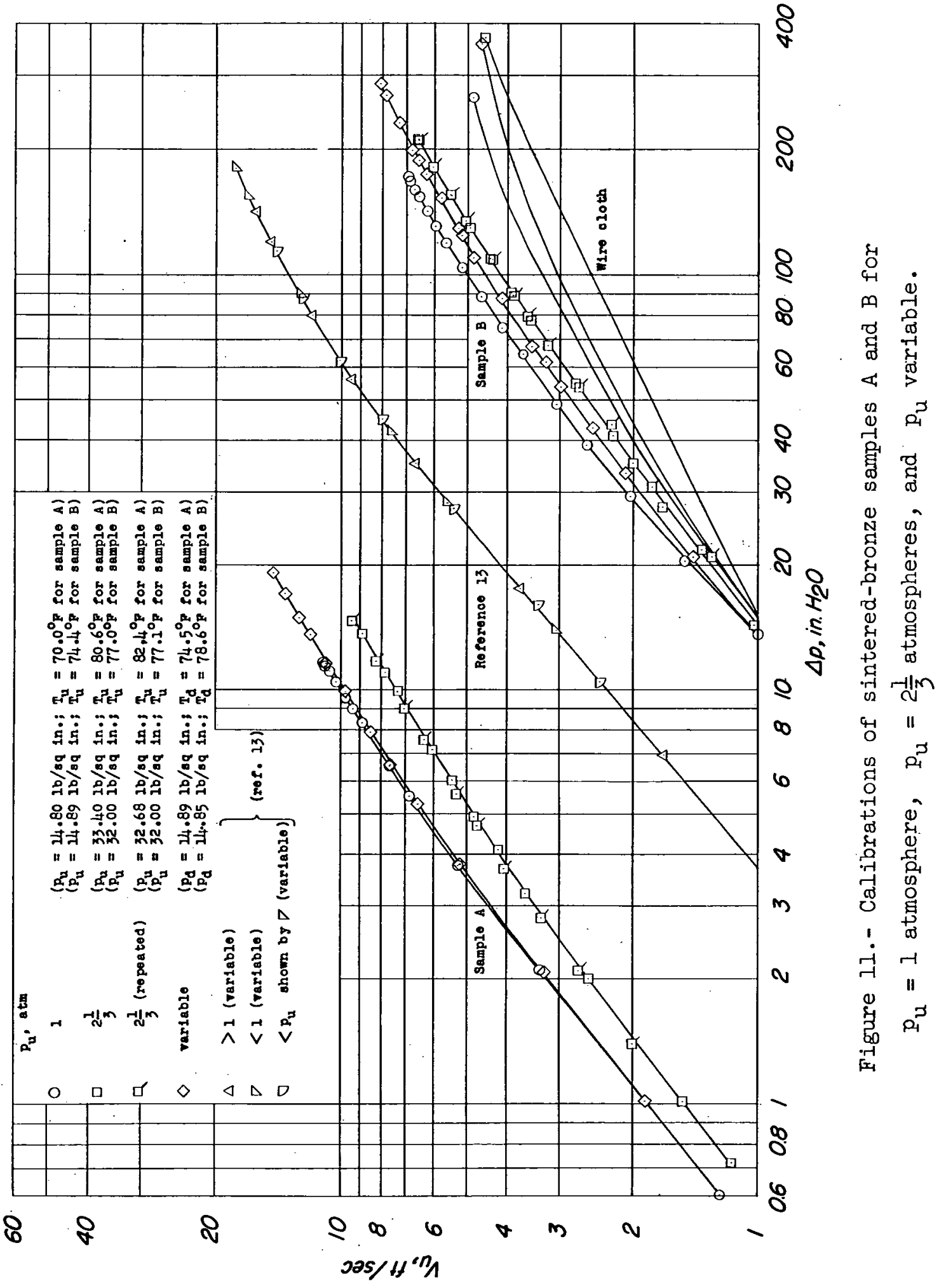



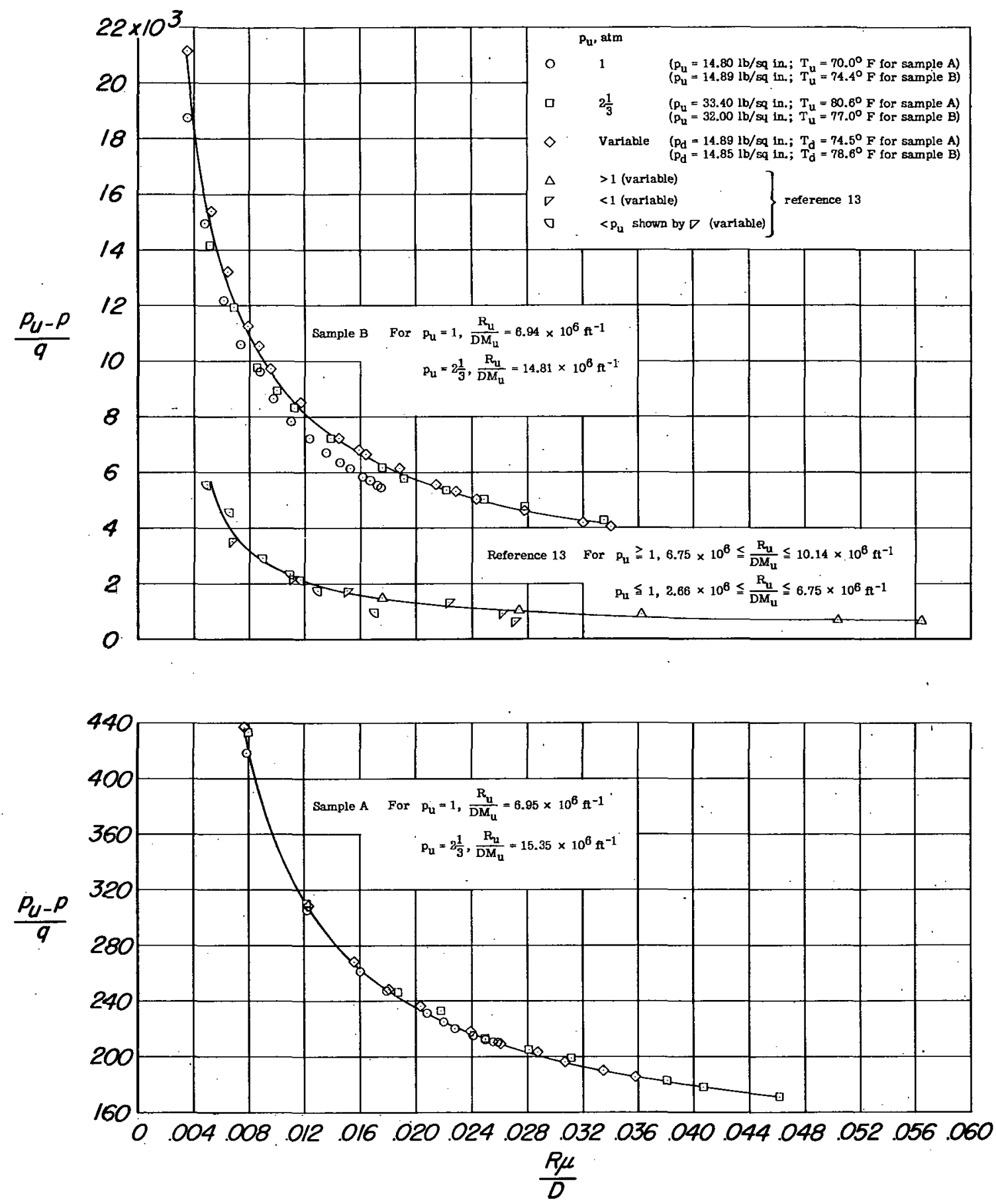

Figure 12.- Test for consistency of data for sintered-bronze samples A and $B$ and for reference 13 . 

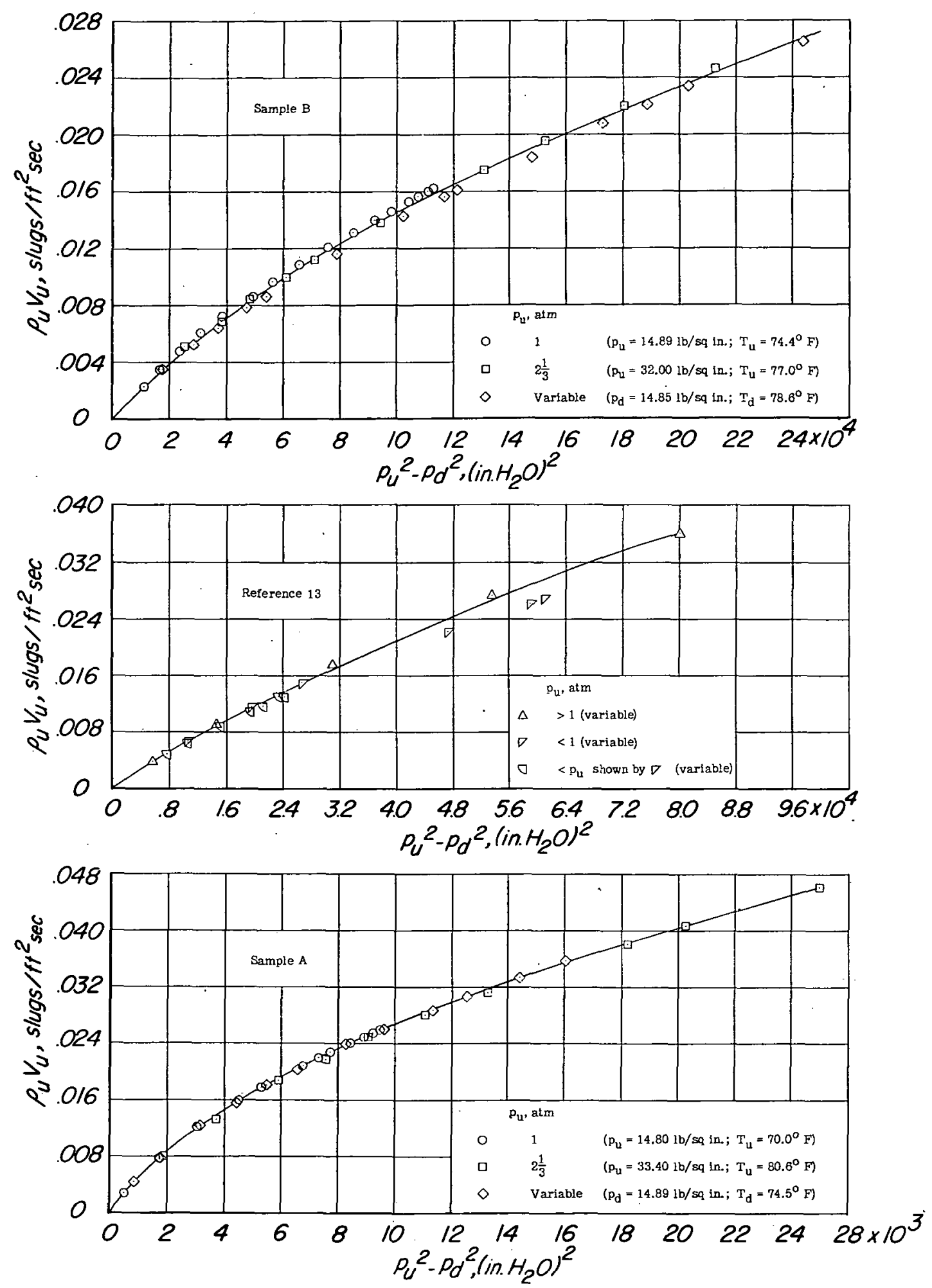

Figure 13.- Correlation of sintered-bronze calibrations based on Darcy's law. 


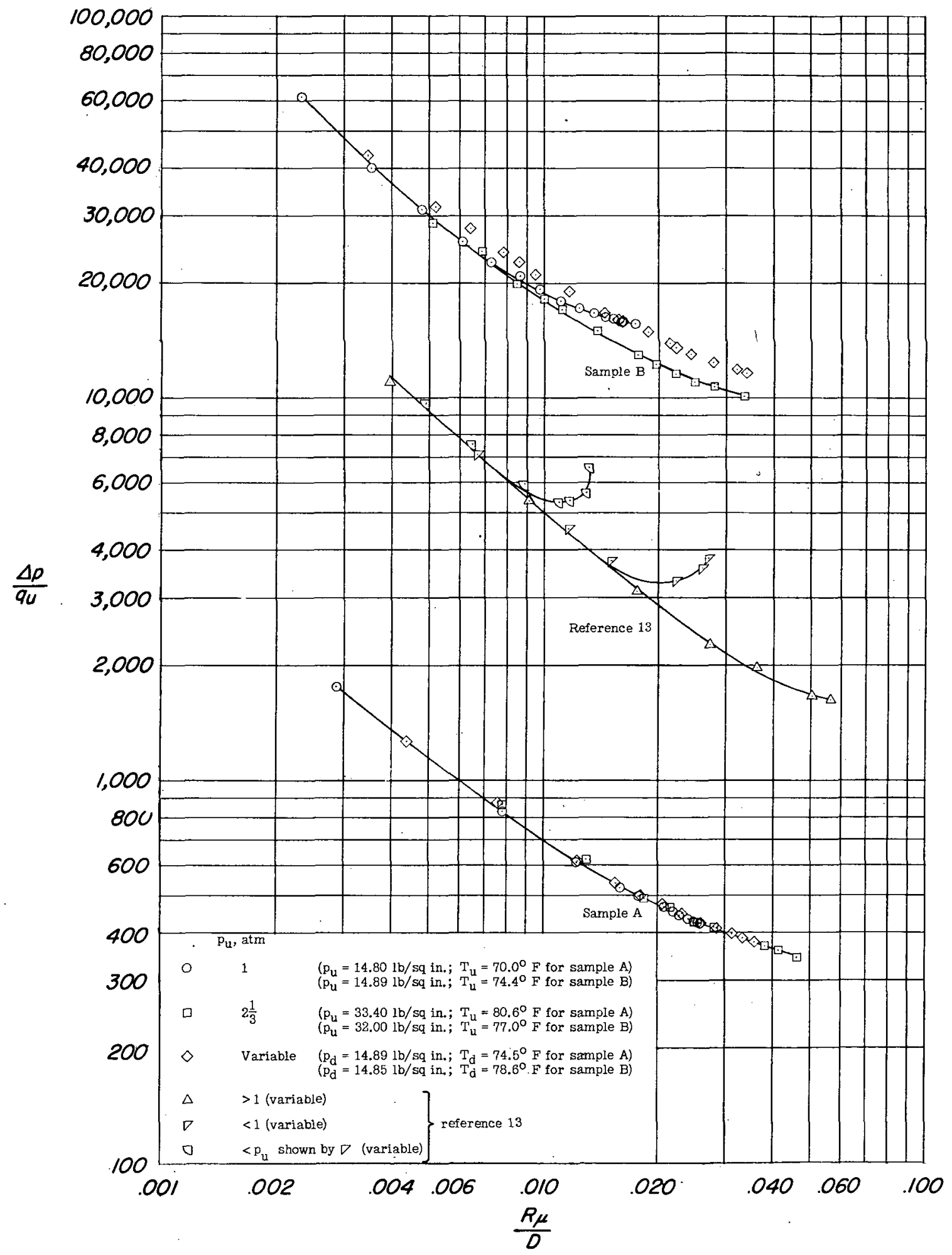

Figure 14.- Variation of $\Delta p / q_{u}$ with $R_{\mu} / D$ for sintered bronze. 